\title{
Minimally invasive approach for treating voluminous periapical cysts of the frontal maxillary teeth
}

\author{
Raluca A. Dragomir' ${ }^{1}$, Andrada R. Doscas' ${ }^{1}$, Florin Sava², \\ Andrei Nicolau', Victor-Vlad Costan ${ }^{1}$ \\ ${ }^{1}$ Oral and Maxillofacial Surgery Department, "Grigore T. Popa" University of Medicine and Pharmacy, \\ Iasi, Romania \\ ${ }^{2}$ Oral and Maxillofacial Surgery Clinic, "St. Spiridon" Hospital, lasi, Romania
}

\begin{abstract}
The aim. Treatment of a large cystic lesion in the maxillary bone involving the nasal cavity and the sinus may be challenging. The traditional approach represented by simple enucleation could irreversibly damage the anatomical structures, therefore, we aimed to evaluate the value of decompression prior to the actual surgical procedure.

Materials and methods. Our treatment strategy focused first on minimizing the lesion by decompression and then performing enucleation.

Results. Follow-up cone beam computed tomography scans showed progressive bone healing and the treatment results were satisfying, with low morbidity rate and no recurrence.
\end{abstract}

Keywords: periapical cyst, nasal cavity, maxillary sinus,

decompression, cone beam computed tomography

\section{INTRODUCTION}

Periapical cysts are frequent lesions encountered in daily practice that arise from the necrotic remnants of the dental pulp. Among all odontogenic jaw cysts, the periapical cyst is the most frequent form, reaching approximately $62 \%$ of all cysts (1). The periapical cyst has male predilection and occurs more often in the permanent dentition than in the temporary one (incidence rate $68 \%$ versus $3.3 \%)(2)$.

It usually grows slowly, commonly asymptomatic and discovered during routine radiographic examination. However, when it reaches a large size it can cause destruction of the surrounding structures, tooth mobility and/or displacement, and root resorption of the adjacent teeth.

The majority of the radicular cysts $(60 \%)$ are seen in the upper jaw and because of the bone structure, it is easier for the lesion to occupy space and expand towards the nasal cavity or maxillary sinus $(3,4)$. For large lesions, it is important to carefully plan the surgical approach. The choice of treatment must be guided by factors such as the extension of the lesion, the relation with anatomical structures, the evolution, the clinical characteristics of the lesion, and nevertheless the systemic condition of the patient (5). The aim of this study was to evaluate the efficacy of decompression as a first stage procedure in order to facilitate the future cyst enucleation.

\section{MATERIAL AND METHODS}

We performed a 4-year retrospective analysis of the records of 8 patients, who presented large cystic lesions intruding the nasal cavity and the maxillary sinus with origin in a frontal tooth. All patients underwent Cone Beam Computed Tomography (CBCT) and dental examination for precise diagnosis of the location and extent of the lesions. The patients' clinical data, including sex, age, medical record and dental focus were analyzed. For all patients, the surgeons opted for a 2-stage surgery protocol: decompression followed by cyst enucleation. 


\section{RESULTS}

A total of 8 cases of large periapical cysts extended to the nasal cavity and maxillary sinus were analyzed over a 4-year period. The study population comprised 2 women and 6 men, with a mean age of 41 years old. The lesions mostly occurred in the 3 rd decade of life.

A total of 28 teeth were affected, the most commonly involved being the lateral incisor followed by the canine. Deciduous teeth were not involved in any case. Related to the causal dental pathology, in two cases the origin was due to retained roots and in six patients there were teeth with endodontic treatment failure. There were no cases of residual cysts.

Imaging examination revealed large cystic lesions extending towards surrounding structures: the nasal cavity, hard palate and the maxillary sinus (Fig. 1, 2).

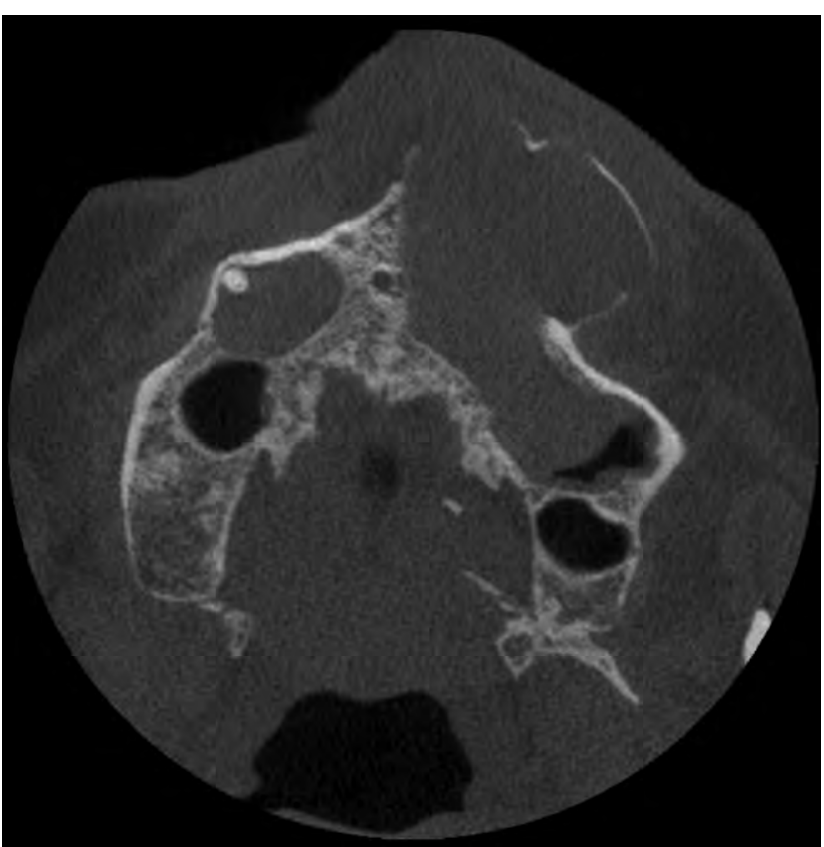

FIGURE 1. Axial cone beam computed tomography scan shows a radio-opaque area involving the upper maxillary alveolar process and the left maxillary sinus with the forward expansion of its anterior wall

The first stage surgery was represented by decompression using a silicone tube, with concurrent biopsy of the cystic membrane (Fig. 3). Following decompression, patients were instructed to irrigate the cavity with saline solution twice a day.

Follow-up CBCT exam after decompression showed progressive bone healing (Fig. 4, 5). After a mean period of 6 months, second stage surgery was performed and was represented by enucleation with direct suture closure in 6 cases and by cystectomy with wound packing in 2 cases (Fig. 6, $7)$. In the cases in which packing of the cavity was performed, an obturator prosthesis was designed (Fig. 8, 9) after wound epithelization, in order to partially fill the cyst cavity and protect it from food debris until complete healing and remodeling of the residual bone. Patients were monitored 6 months after surgery, showing a favorable evolution with no sign of recurrence.

\section{DISCUSSIONS}

The periapical cyst develops from remnants of Malassez epithelium, which proliferates and forms the cystic wall (6). The expansion takes place through several mechanism: the destruction of extracellular matrix by collagenase on one hand and by bone resorption determined by osteoclastlike cells on the other hand (7).

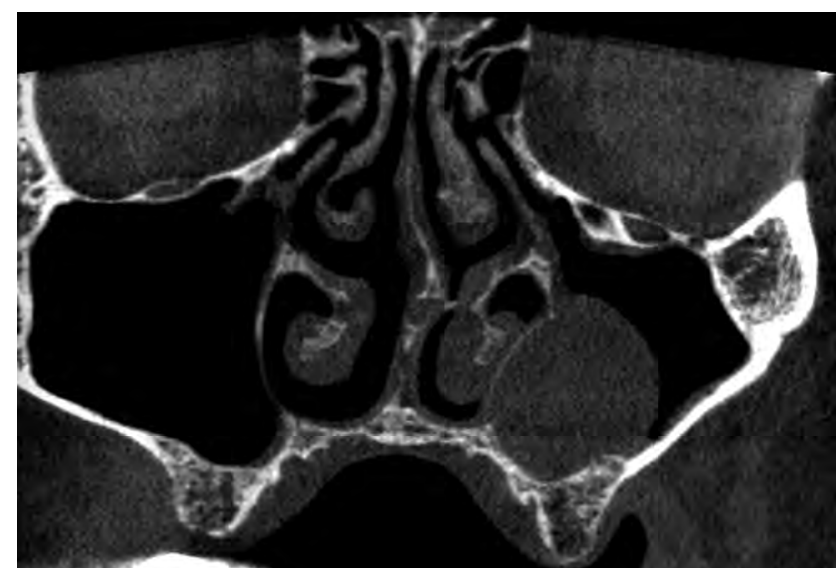

FIGURE 2. Coronal cone beam computed tomography scan before surgical treatment, showing a radio-opaque area involving the left maxillary sinus and the inferior meatus of the nasal cavity

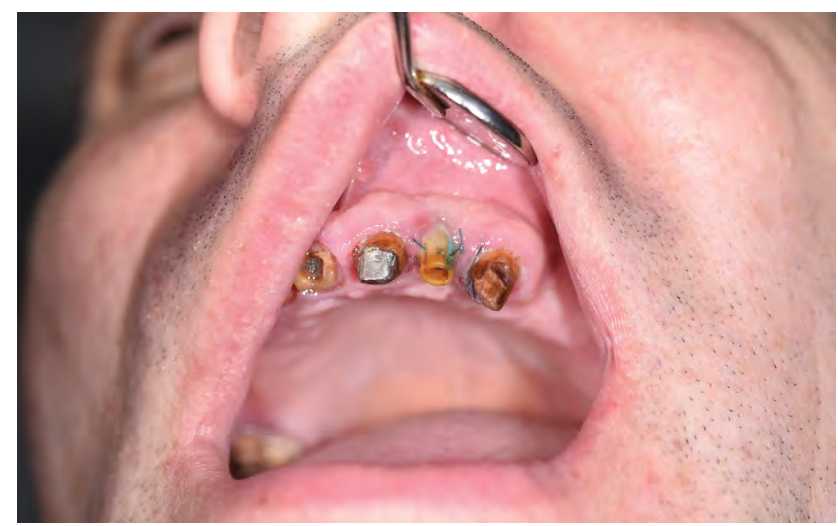

FIGURE 3. Decompression using a silicone tube placed in the post-extraction socket of tooth 22 


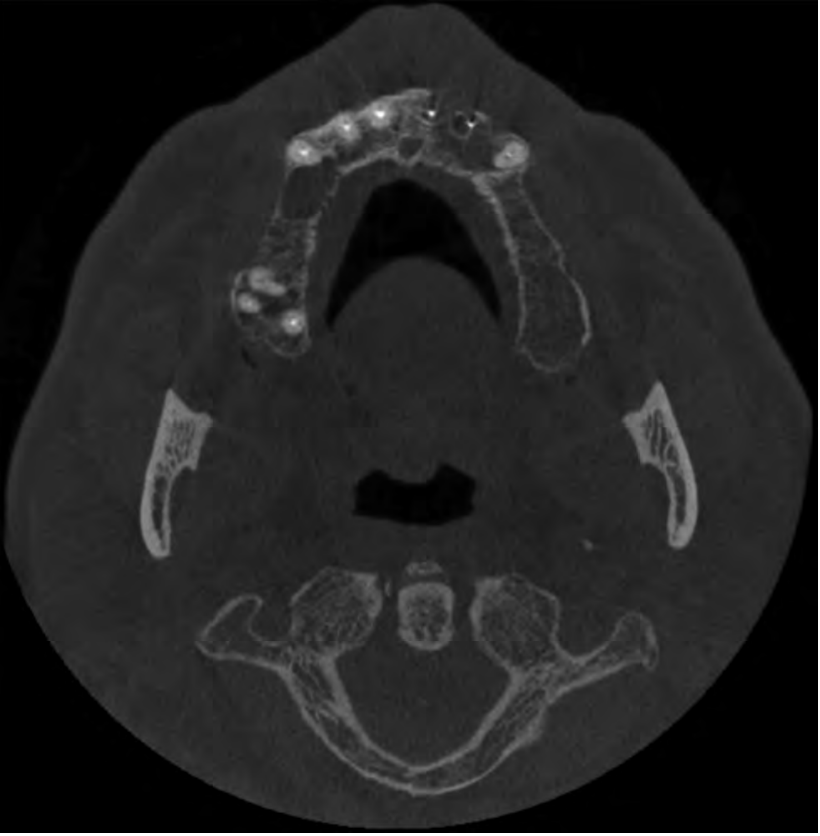

FIGURE 4. Axial cone beam computed tomography image shows the reduction in size of the lesion 8 months after decompression

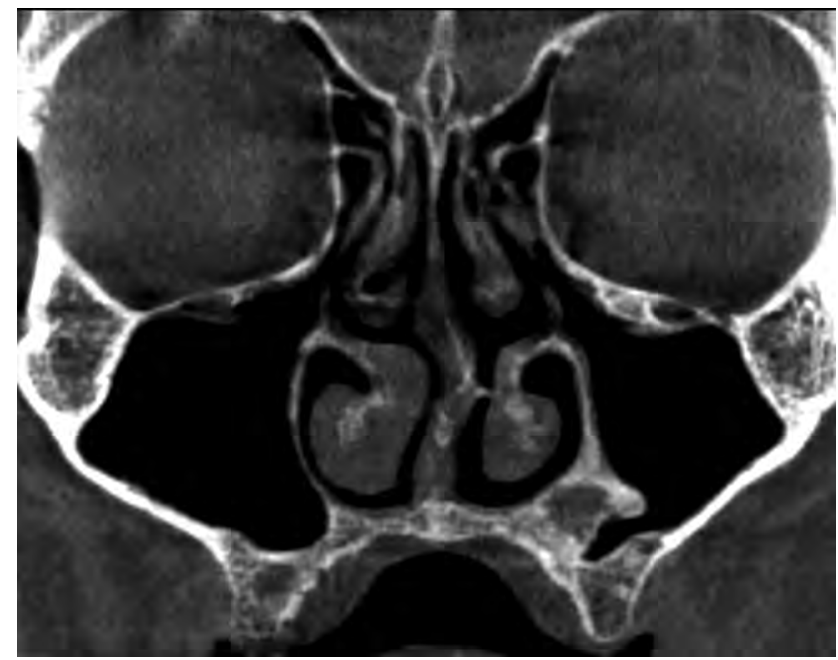

FIGURE 5. Coronal computed tomography image, 8 months after decompression, showing the recovery of the anatomical architecture of both maxillary sinus and the nasal cavity

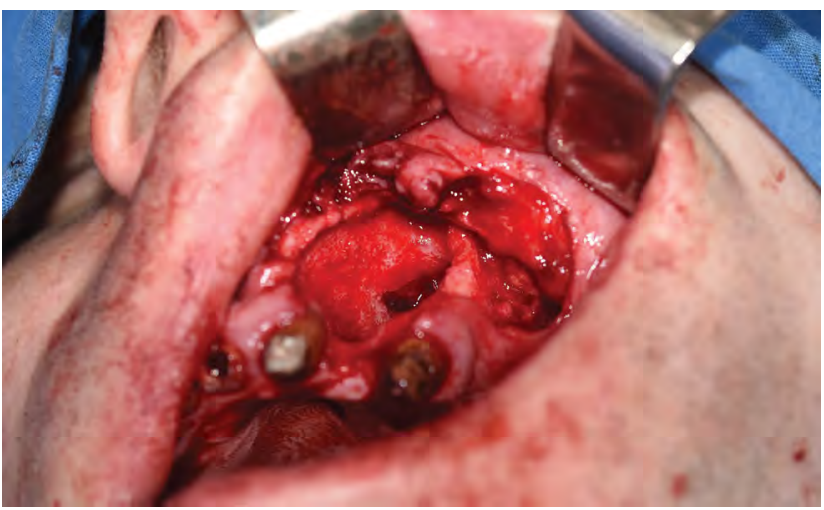

FIGURE 6. Intraoperative aspect of the cyst cavity following cystectomy

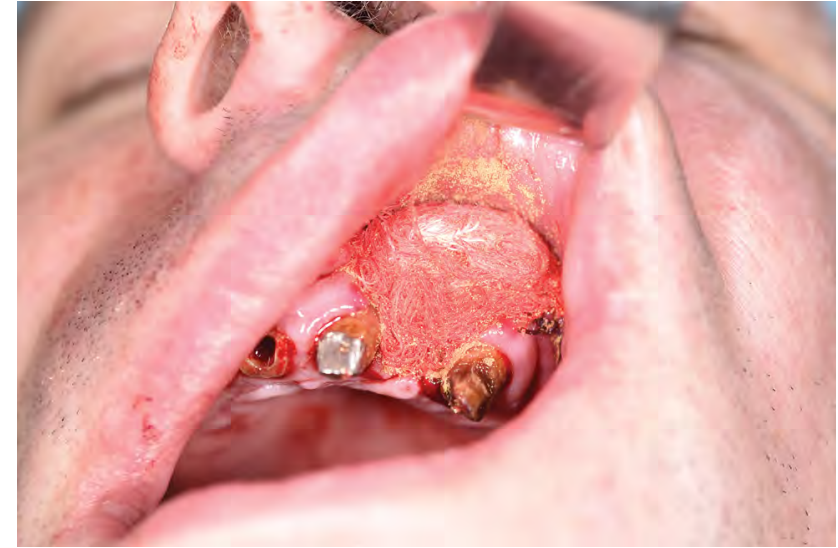

FIGURE 7. Wound packing with iodoform gauze over the vestibular mucosal flap at the end of the cystectomy procedure

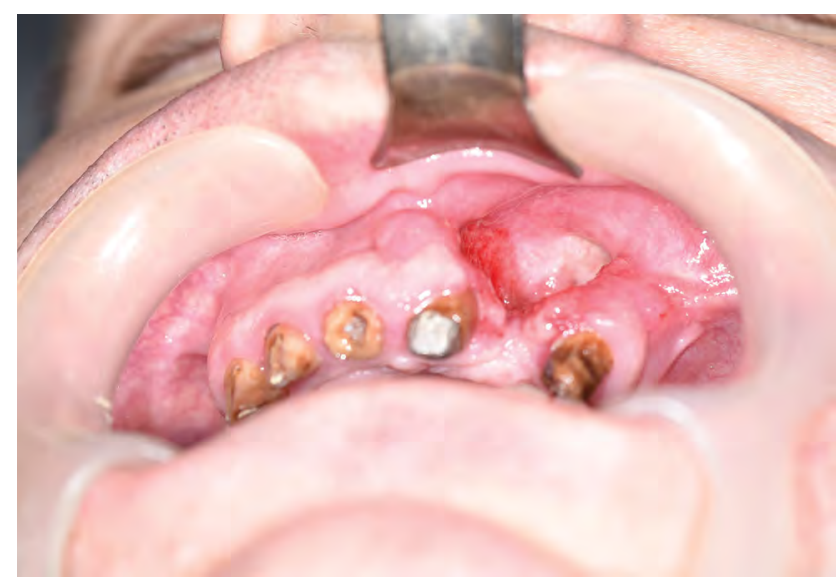

FIGURE 8. Postoperative aspect of the epithelized cavity three weeks after the cystectomy procedure

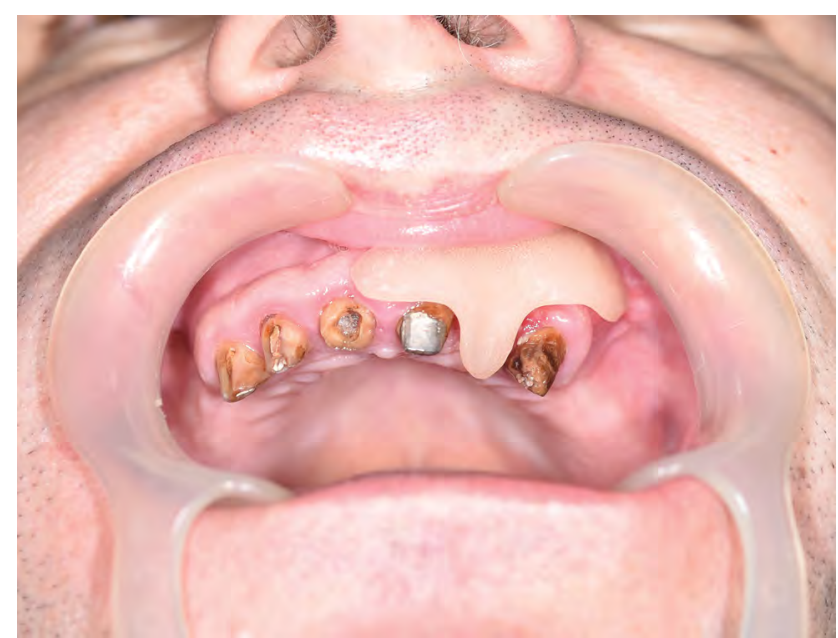

FIGURE 9. Obturator prosthesis partially protecting the cystic cavity until complete healing and tissue remodeling

Also, interleukin-1 $\alpha$ (IL-1 $\alpha)$ and interleukin 6 (IL-6) are thought to be involved in cyst expansion because the intra-cystic pressure induces the release of these cytokines which stimulate bone resorption by activating the osteoclast cells (8). 
Radicular cysts are usually asymptomatic except the cases of acute infection when pain, swelling and fistulas can develop. The diagnosis is based on clinical findings (nonvital teeth, bone expansion) and radiological explorations but it is confirmed by histological analysis. When cysts are large, CBCT scan is preferred over panoramic radiographs because it provides superior details, in terms of size and extent of the lesion towards vital anatomical structures. Nasal cavity and the maxillary sinus are vulnerable structures that can be invaded by odontogenic cystic lesions due to their close relation with the maxillary alveolar bone.

Large cyst developed in the anterior part of the maxillary bone are very challenging to manage because of their close relation with both anatomical structures: the nasal cavity and the maxillary sinus. A classical treatment represented by enucleation could be followed by several complication, the oralnasal communication being one of the most fearful because the available techniques for reconstruction are sensitive, demanding, with high risks of recurrence. As an alternative, decompression represents a minimally invasive approach with the aim to shrink the volume of the cyst and its closeness to anatomical structures and to allow a second stage surgery represented by the removal of the residual lesion. Decompression of a fluid containing cyst can only be achieved if the fluid is not allowed to reaccumulate within it. Through effective cyst decompression, a significant change in the hydrostatic pressure occurs, allowing the lesion to shrink instead of expanding (9). The advantage of this procedure is that it minimizes the damage of the tissues and that it reduces bone resorption and stimulates osteogenesis by inhibiting IL-1 expression (10).

Decompression was preferred for our patients and after the cystic lesions decreased in size, enucleation was performed. The purpose of this procedure was to eliminate the epithelial remnants of the cystic membrane. Because of the decreased size of the cyst and thickened bone, the curettage of bone of the cystic cavity was achieved without damaging the nasal structures or sinus wall destruction.

A large maxillary cyst that involves the entire sinus cavity can determine nasal and ophthalmological symptoms: exophthalmos, epiphora, nasal obstruction or nasal discharge (11). For these types of lesions, the traditional Caldwell-Luc procedure represents a wide approach for a complete excision, but it is invasive and causes postoperative discomfort such as swelling, hematoma and, frequently, damages the bone structures and the ciliary mucosa. Potential complications of this technique include oro-antral fistulas formation, infection, teeth loss, scars and the necessity of reconstructive surgery $(12,13)$. Moreover, the epithelializing of the sinus cavity walls is achieved by the oral mucosa, compromising the normal mucociliary physiology leading to the sinus clearance impairment with the risk of mucocele development or of chronic osteitis (14-16). Despite the fact that is a time-consuming method and requires patients' compliance, decompression is preferred over invasive methods, because it not only offers the possibility to preserve bone architecture, but it also facilitates the future surgical procedure.

The endonasal endoscopic approach is another method used for complete removal• of cysts with maxillary sinus involvement (17). Marsupialization of any cyst involving the sinus cavity with a nasal endoscopic approach is a diagnostic tool and therapeutic alternative to the transoral approach (18). It is considered that it is less time consuming, postoperative discomfort is absent and it has no risk of recurrence (18). Despite all of the advantages of endoscopic surgery, we consider that the content of cysts can be drained much more easily into the oral cavity, according to the laws of gravity. Therefore, the oral approach is more preferable than the nasal one.

\section{CONCLUSIONS}

In cases of extensive cysts, in order to avoid possible future complications and to respect the normal architecture of the surrounding tissues, the treatment strategy focuses first on minimizing the lesion by decompression and later on performing enucleation. Cone beam computed tomography is preferred because the surgeon can assess the exact extension of the lesion, plan the surgical protocol and monitor the results. 


\section{REFERENCES}

1. Neville BW, Damm DD, Allen CM, Bouquot JE. Oral and Maxillofacial Pathology 2nd Edition. Saunders: An imprint of Elsevier, 2005.

2. Krishnamurthy V, Haridas $S$, Garud M. Radicular cyst masquerading as a multilocular radiolucency. Quintessence Int. 2013; 44: 71-73.

3. Shear M, Speight P. Cysts of the Oral and Maxillofacial Regions. Oxford: Wiley-Blackwell, 2007.

4. Pekiner FZ, Borahan O, Ugurlu F. Clinical and radiological features of a large radicular cyst involving the entire maxillary sinus. MUSBED. 2012; 2: 31-36.

5. Ribeiro, Paulo Domingos Jr. et al. Surgical approaches of extensive Periapical cyst. Considerations about Surgical technique, 2004; 23(2): 317-328.

6. Meghij S, Yureshi W, Henderson B et al. The role of endo- toxin and cytokines in the pathogenesis of odontogenic cysts. Arch Oral Biol, 1996; 41: 523-551.

7. Kubota $\mathrm{Y}$, Imajo I, Itonaga $\mathrm{R}$ et al. Effects of the patient's age and the size of the primary lesion on the speed of shrinkage after masupialisation of keratocystic odontogenic tumours, dentigerous cysts, and radicular cysts. Br J Oral Maxillofac Surg. 2013;51: 358-362.

8. Mudjono H, Rahajoe PS, Hasan CY. Marsupialization of radicular cysts on adolescent patients to reduce treatment morbidity (case report). J Dentomaxillofac Sci. 2017; 2(2):139-142.

9. Pogrel MA. Treatment of keratocysts: the case for decompression and marsupialization. J Oral Maxillofac. 2005;63(11):1667-1673.

10. Nanomiya T, Kubota $Y$, Koji T et al. Marsupialisation inhibits interleukin-1 alpha expression and epithelial cell proliferation in odontogenic keratocysts. J Oral Pathol Med. 2002;31: 526-533.
11. Bouguezzi Adel, Souid Kawthar, Boudegga Souha, Boughzala Abdellatif. Extensive periapical cyst in the maxillary sinus: A case report. Int Dental J Stud Res. 2012. Available at http://www.idjsr.com/ uploads/21/1265_pdf.pdf.

12. Ozer $\mathrm{S}$, Cabbarzade $\mathrm{C}$, Ogretmenoglu O. A new transnasal approach to naso-labial cyst: endoscopic excision of naso-labial cyst. $J$ Craniofac Surg. 2013; 24: 1748-1749.

13. Huang CC, ChenCW ,LeeTJ ,ChangPH ,ChenYW , ChenYL ,Fu CH, Wu CC, Huang CC. Transnasal endoscopic marsupialization of postoperative maxillary mucoceles: middle meatal antrostomy versus inferior meatal antrostomy. Eur Arch Oto-Rhino-Laryngol. 2011; 268:1583-1587.

14. Hajiioannou J, Koudounarakis E, Alexopoulos K, Kotsani A, Kyrmizakis DE. Maxillary sinusitis of dental origin due to oroantral fistula, treated by endoscopic sinus surgery and primary fistula closure. J Laryngol Otol. 2010;124:986-989.

15. Govindaraj S, Adappa ND, Kennedy DW. Endoscopic sinus surgery: evolution and technical innovations. J Laryngol Otol. 2010; 124:242-250.

16. Sawatsubashi M, Murakami D, Oda M, Komune S. Transnasal endoscopic surgery of post-operative maxillary cysts. J Laryngol Otol. 2015;129(Suppl 2):S46-51.

17. Sagit M, Guler S, Tasdemir A, Akf Somdas M. Large radicular cyst in the maxillary sinus. J Craniofac Surg. 2011; 22:64.

18. Consolo U, Bellini P, Lizio G. Trans-nasal endoscopic marsupialization of a voluminous radicular cyst involving maxillary sinus and nasal cavity: A case report and a review on this surgical approach. J Oral Maxillofac Cases. 2018; 91-96. 